\title{
APLICAÇÃO DE MÉTODOS DE LUMINESCÊNCIA E DA RESSONÂNCIA DE SPIN ELECTRÓNICA NA DATAÇÃO DE UNIDADES SEDIMENTARES QUATERNÁRIAS E DA EVOLUÇÃO DAS PAISAGENS
}

\author{
Pedro P. Cunha ${ }^{(a)}$, António A. Martins ${ }^{(b)}$ \\ (a) MARE - Centro de Ciências do Mar e do Ambiente; Departamento de Ciências da Terra da Universidade de \\ Coimbra; pcunha@dct.uc.pt \\ (b) Instituto de Ciências da Terra; Departamento de Geociências, Universidade de Évora, aam@uevora.pt
}

Eixo: GEOCRONOLOGIA E ESTUDOS PALEOAMBIENTAIS

\section{Resumo}

Apresenta-se uma síntese dos princípios, métodos e aplicações da datação por luminescência a estudos de Geomorfologia ou de Geologia do Quaternário, bem como alguns exemplos da datação de depósitos sedimentares portugueses.

Palavras chave: Geomorfologia, estratigrafia, datação por luminescência, dose de radiação ambiental, dose equivalente.

\section{USE OF THE LUMINESCENCE AND ELECTRON SPIN RESONANCE METHODS FOR THE DATING OF QUATERNARY SEDIMENTARY UNITS AND LANDSCAPE EVOLUTION}

\begin{abstract}
A synthesis of the principles, methods and applications of the luminescence dating for Geomorphology and Quaternary Geology studies, but also some examples of dating Portuguese sedimentary deposits are here presented.
\end{abstract}

Keywords: Geomorphology, stratigraphy, luminescence dating, dose-rate, equivalent dose.

\section{Introdução}

A determinação de idades por luminescência e por ressonância electrónica de spin tem tido um desenvolvimento exponencial na última década, permitindo estabelecer a idade de sedimentos, materiais arqueológicos líticos ou de cerâmicas, bem como superfícies de pedra, que se encontravam enterrados após uma eficaz exposição à luz ou ao calor.

Uma das grandes vantagens da utilização destes processos de datação absoluta é que podem ser utilizadas para intervalos que abranjam poucas décadas até várias centenas de milhares de anos (luminescência), ou 
mesmo centenas de milhares de anos a alguns milhões de anos), necessitando da medição em quartzo ou feldspato potássico (principais minerais usados como dosímetros).

A necessidade de constringir no tempo registos sedimentares do Quaternário e os eventos geológicos que eles representam tem levado os pesquisadores a incluir datações absolutas nos seus estudos.

Embora com as problemáticas associadas à correção do efeito "reservatório" para sedimentos de transição, da possibilidade de reciclagem de matéria orgânica antiga e do seu limite máximo de aplicabilidade a depósitos com idade até ca. $50 \mathrm{ka}$, a datação por $\mathrm{C}^{14}$ tem sido extensivamente usada. Contudo, para sedimentos mais antigos ou sem matéria orgânica, é necessário fazer a datação absoluta por outros métodos.

O método por U-Th permite a datação de carbonatos, ossos e dentes fósseis, cuja idade esteja compreendida entre 10 e 350 ka. No caso dos ossos e dentes fósseis, o urânio só penetra neles depois da morte e enterramento dos animais e pode acontecer de várias formas (precoce ou progressiva), o que representa uma limitação na confiabilidade do método. O modelo de incorporação precoce dá uma idade mínima à amostra.

\section{Princípios da datação por luminescência e por ressonância electrónica de spin}

Os métodos de datação por luminescência e por ressonância electrónica de spin são usados para determinar o tempo decorrido desde que:

- Um sedimento foi pela última vez exposto à luz;

- Ocorreu o último aquecimento significativo de cerâmicas ou clastos (ex. em fogueiras).

Quando grãos minerais estão enterrados, recebem radiação ionizante proveniente de elementos radioactivos (ex. Th, $\mathrm{U} \mathrm{e}^{40} \mathrm{~K}$ ) existentes nos sedimentos próximos, mas também da radiação cósmica, que tem alguma penetração em profundidade.

A interação das radiações ionizantes naturais com a matéria cristalina gera carga eléctricas que ficam retidas em armadilhas no edifício cristalino de minerais (ex. quartzo, feldspato) e constituem a Paleodose.

É crucial que antes do enterramento de um sedimento, ele seja convenientemente exposto à luz solar pois esta descarrega a energia latente anterior, processo designado por branqueamento (bleaching). Permite que o "relógio" da contagem do tempo seja colocado a zero; caso contrário, a idade da amostra ficará sobrestimada. O incompleto branqueamento pode ser significativo em sedimentos do Holocénico, mas tem pouca relevância em sedimentos do Plistocénico, pois nestes a energia presente na altura do enterramento é, geralmente, muito pequena relativamente à acumulada após a última exposição à luz. Sedimentos coluvionares, glaciários e aluviais apresentam muito maior tendência para incompleto branqueamento do que os depositados em ambientes fluviais, litorais ou eólicos. 


\section{OS DESAFIOS DA GEOGRAFIA FÍSICA NA FRONTEIRA DO CONHECIMENTO \\ Instituto de Geociências - Unicamp \\ Campinas - SP \\ 28 de Junho à 02 de Julho de 2017}

Em laboratório, obtém-se a Dose equivalente (De), expressa em Gray (Gy), pretendendo esta representar a

Paleodose. Na sua estimação os grãos minerais habitualmente usados como dosímetros são o quartzo (Qz) e o feldspato potássico (Fk). A idade de uma amostra obtém-se dividindo a Dose equivalente pela Taxa de radiação ambiental (dose-rate), expressa em Gy/ka (Fig. 1).

A taxa de radiação ambiental pode ser medida no campo, através de um espectómetro gama de baixa resolução, mas é recomendável que seja medida em laboratório por meio de um espectómetro gama de alta resolução. Este quantifica os elementos radioactivos, permitindo saber se houve manutenção da relação entre isótopos-pai e isótopos-filhos durante o período de enterramento; caso contrário, a taxa de radiação ambiental não esteve constante e o valor actual não representa o valor médio (e não se pode usar para determinar a idade real da amostra). Quando a medição da taxa de radiação é feita em laboratório é necessário que a subamostra medida seja representativa de um volume esférico com cerca de $0,5 \mathrm{~m}$ de diâmetro que no campo envolvia a amostra recolhida; caso contrário, a taxa de radiação medida no laboratório não é igual à que afectou a amostra recolhida (e a idade fica subestimada ou sobreestimada). Quando se pretende datar uma camada pouco espessa de sedimento com taxa de radiação diferente da dos sedimentos enquadrantes (por exemplo uma camada de $10 \mathrm{~cm}$ de areia de tsunami intercalada em siltes estuarinos) é necessário fazer a modelização espacial da dose de radiação.

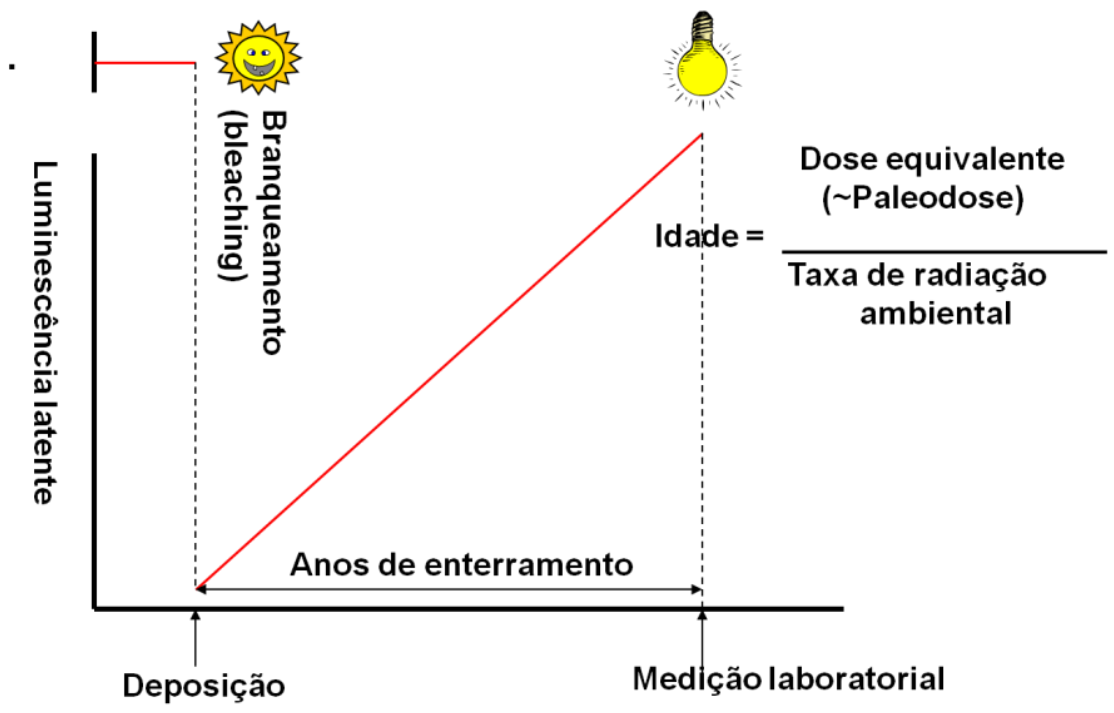

Figura 1 - Esquema ilustrativo da determinação de idade por luminescência e por ressonância electrónica de spin.

Um outro parâmetro que influencia o cálculo da taxa de radiação é o teor em água do sedimento e a quantidade de cimento carbonatado, pois estes reduzem a penetração da radiação (e a resultante ionização). Assim, deve-se determinar o teor de água no momento de colheita da amostra e o valor em 
saturação; com base nestes valores e tendo em conta a provável posição do nível freático durante o período de enterramento, estima-se o valor adequado a inserir no cálculo da taxa de radiação ambiental. Desta forma, a dose de radiação (energia absorvida por ano) por um sedimento enterrado resulta de:

- Radionuclídeos internos - fornecendo a dose de radiação interna beta (e alpha) ( $30 \%$ do total para um feldspato-K, negligível em quartzo);

- Radionuclídeos externos - fornecendo dose de radiação externa alpha, beta e gamma, a partir da matriz envolvente. A concentração em carbonatos e o teor em água (teor de campo, teor de saturação, estimativa para o tempo de enterramento, tendo em conta a provável posição do nível freático) afectam o resultado da idade até $10 \%$;

- Radiação cósmica, uma pequena \% do total, e que se atenua rapidamente em profundidade.

\section{Metodologias para datação por luminescência e por ressonância electrónica de spin}

Actualmente são várias as metodologias usadas rotineiramente na datação por luminescência, e muitas outras se encontram em desenvolvimento:

- Termoluminescência (TL; Thermo-Luminescence), havendo emissão de luz induzida por aquecimento;

- Luminescência Opticamente Estimulada (LOE; OSL - Optically Stimulated Luminescence), havendo emissão de luz induzida por estimulação de fotões; o método de rotina é por Qz-OSL;

- Infra Red Stimulated Luminescence (IRSL), aplicável a feldspato-K mas acarretando uma correcção de uma perda de energia com o tempo (anomalous fading);

- Sinal post-IR IR, metodologia nova aplicável a feldspato-K e não tendo anomalous fading;

- TT-OSL, etc.

As datações por OSL estão muito mais generalizadas que por TL. Isto deve-se ao facto do sinal luminescente resultante da estimulação pela luz ser mais rapidamente e eficazmente limpo" (branqueado) do que o sinal resultante da estimulação pela temperatura, sendo essa uma das principais vantagens da OSL relativamente à TL. Além disso, as fracções medidas por TL são feitas em grãos muito finos (10-4 um), pelo que a sua composição pluri-mineral também não permite as necessárias correções às medições e também, porque existem sedimentos desprovidos dessa fraçcão de silte fino (ex. areias de praia ou eólicas). 


\section{Amostragem para datação por luminescência ou para datação por ressonância} electrónica de spin

A estratégia da amostragem deve ser previamente estabelecida em função dos objectivos do estudo e do financiamento disponível para ser gasto em datações. Sedimentos ricos de quartzo (plutónico ou metamórfico) ou de feldspato-K são possíveis de ser datados por luminescência, enquanto que a ressonância electrónica de spin apena usa grãos de quartzo.

Sedimentos arenosos ou lutíticos, em função da sua consolidação, podem ser amostrados com tubos opacos de plástico (PVC) ou metálicos (em geral com 6-8 cm de diâmetro e $\sim 30 \mathrm{~cm}$ de comprimento). Sedimentos mais grosseiros (cascalhentos) exigem uma adequada metodologia para a determinação da dose de radiação e amostragem. A amostragem também pode ser feita durante a noite, para sacos de plástico pretos espessos (opacos à luz), com o auxílio de lanternas de luz vermelha fraca. Sedimentos consolidados por cimentação carbonatada exigem a destruição desse cimento sob condições laboratoriais com iluminação adequada para não se afectar a paleodose dos grãos a medir. Também se podem datar sedimentos de sondagens, com recolha em tubo opaco (ex. para datar sucessões holocénicas estuarinas ou dunares).

A amostragem para datação por luminescência ou ESR deve ser feita por investigador com experiência, para se evitarem contextos impróprios de recolha. Nomeadamente, devem-se evitar: a) sedimentos que mostrem perturbações posteriores à deposição e que promovam a entrada de luz (fendas de dissecação, bioturbação e interferência de raízes, revolvimento agrícola, movimentação de vertente, etc.); b) modificações diagenéticas (existência de solo, lavagem pela água de infiltração, formação e transporte de matriz argilosa, migração de isótopos radioactivos, etc.); c) heterogeneidades espaciais da radiação ambiental. Colheitas impróprias acarretam que se meçam no laboratório doses-equivalentes e doses de radiação incorrectas, produzindo-se idades diferentes dos eventos reais a datar.

\section{Preparação de fracções minerais em sala com luz "vermelha"}

A preparação de fracções minerais para medição OSL ou ESR efectua-se em sala com iluminação específica ("vermelha") e compreende as seguintes fases:

- Abertura das amostras (geralmente tubos opacos);

- Crivagem por via húmida, para se obterem várias fracções dimensionais;

- Geralmente é a fracção 250-180 um que é selecionada para depois ser sujeita a ataques por $\mathrm{HCl}$ (10\%) e $\mathrm{H}_{2} \mathrm{O}_{2}(10 \%)$; lavagens com $\mathrm{H}_{2} \mathrm{O}$;

- Secagem em estufa a $40^{\circ} \mathrm{C}$.

- Separação das fracções de quartzo e feldspato-K por líquido com densidade 2,56; 
- Ataque com $\mathrm{HF}, \mathrm{HCl}$ e lavagens das fracções minerais;

- Secagem e acondicionamento das fracções minerais puras (ex. quartzo, feldspato-K);

- Em equipamento leitor de luminescência, medição de De em aliquotes com diferentes quantidades de grãos de um mineral e realização de vários testes.

\section{Alcance temporal da datação por luminescência ou da datação por ressonância electrónica de spin}

A principal limitação do quartzo nas datações por OSL é a saturação dos grãos, que ocorre a 200 Gy. Assim, na medição de grãos de quartzo usando o protocolo SAR (Murray \& Wintle, 2000), a OSL permite datar sedimentos até cerca de 140 ka caso a taxa de radiação ambiental seja inferior a 2 Gy.

Areias e siltes do Holocénico podem ser datados com precisão por OSL em Qz e, consequentemente, servir para determinar a cronologia de eventos geológicos recentes e calcular taxas de sedimentação.

Também se podem usar sedimentos de idade já conhecida - ex: depósitos do tsunami de 1755 (Cunha et al., 2010) ou da cheia de 1909 do rio Tejo - para aferir o processo de medição laboratorial ou determinar a importância do incompleto branqueamento nos ambientes com transporte aquoso turbulento.

Idades da ordem da dezena de anos, por exemplo em areias eólicas, podem ser obtidas usando procedimentos de medição apropriados (Huntley \& Lian, 1999; Wallinga, 2002).

As datações OSL em Qz, apesar de precisas, são praticamente inviáveis para, por exemplo, datar depósitos sedimentares Plistocénicos que possuam altas doses de radiação ambiental, como os de terraços do Baixo Tejo (geralmente 3 a 7 Gy/ka; Martins \& Cunha, 2006).

Luminescência estimulada em infra-vermelho (IRSL; Infra Red Stimulated Luminescence), método que usa o feldspato-K (Fk) como dosímetro. Quando a datação por Qz-OSL só fornece idade mínima (sinal saturado) este método é uma alternativa viável pois a saturação do sinal geralmente só ocorre aos 1000 Gy. Contudo, as idades obtidas por IRSL precisam de ser corrigidas relativamente à perda de energia (anomalous fading) que ocorre nos grãos de $\mathrm{Fk}$ ao longo do tempo (Wintle, 1973) e mudanças de sensibilidade nos defeitos do cristal (Wallinga, 2002); estes dois fenómenos são ainda mal compreendidos e as correções são problemáticas. Infelizmente, na maior parte dos casos, os métodos de correção de fading não são adequados e geram subestimação nas idades obtidas (Cunha, 2010).

Apesar destas dificuldades, foi possível datar por IRSL em feldspato-K os três níveis inferiores de terraços do Tejo português, tendo-se obtido idades corrigidas até 280 ka (Cunha et al., 2008; Martins et al., 2009, 2010a, 2010b). 
O recente protocolo pIRIR290 usa o Fk como dosímetro e uma alta temperatura na estimulação pós-IRIR (Thomsen et al., 2008; Buylaert et al., 2009). Este método constitui a melhor alternativa atual para a datação de amostras que possuem o sinal Qz-OSL em saturação; tem fading irrelevante e pode fornecer resultados precisos até ca. $600 \mathrm{ka}$ (Buylaert et al., 2012).

Muito interessante é a recente possibilidade de se datarem superfícies de rocha (ex. Sohbati et al., 2012).

\section{Avaliação do sinal luminescente residual}

No processo de averiguação do incompleto branqueamento, normalmente reduz-se o número de grãos em cada aliquote (disco contendo grãos, usado para a medição da Dose equivalente) para detectar subpopulações com diferentes De, chegando-se a fazer medições grão a grão. Uma distribuição muito heterogénea da De ou a existência de alguns aliquotes com valores muito superiores aos valores mais frequentes da população são indicadores de incompleto branqueamento (Murray \& Olley, 2002). A presença de sinal latente anterior à deposição tem de ser tomada em conta no cálculo da idade de depósitos modernos.

\section{Exemplo de aplicação datação LOE e ESR a estudos de arquivos do Quaternário e na interpretação da evolução das paisagens do Rio Tejo em Portugal}

A caracterização da escadaria de terraços do Baixo Tejo, na área da bacia sedimentar cenozóica, conheceu um grande desenvolvimento nas duas últimas décadas. Durante este período realizou-se uma cartografia geomorfológica detalhada e obteve-se o conhecimento da posição rigorosa (acima do leito actual) de cada nível de terraço, em diferentes áreas cruzadas pelo rio Tejo (Fig. 2). Em paralelo prosseguiu a caracterização litostratigráfica e sedimentológica dos depósitos de terraço, bem como a utilização de técnicas de datação absoluta, principalmente por U-Th (ex. Raposo, 1995), por LOE (Martins et al., 2009, 2010a, 2010b; Cunha et al., 2008, 2012, 2016, 2017), mas também por ESR (Rosina et al., 2014) (Fig. 3). 

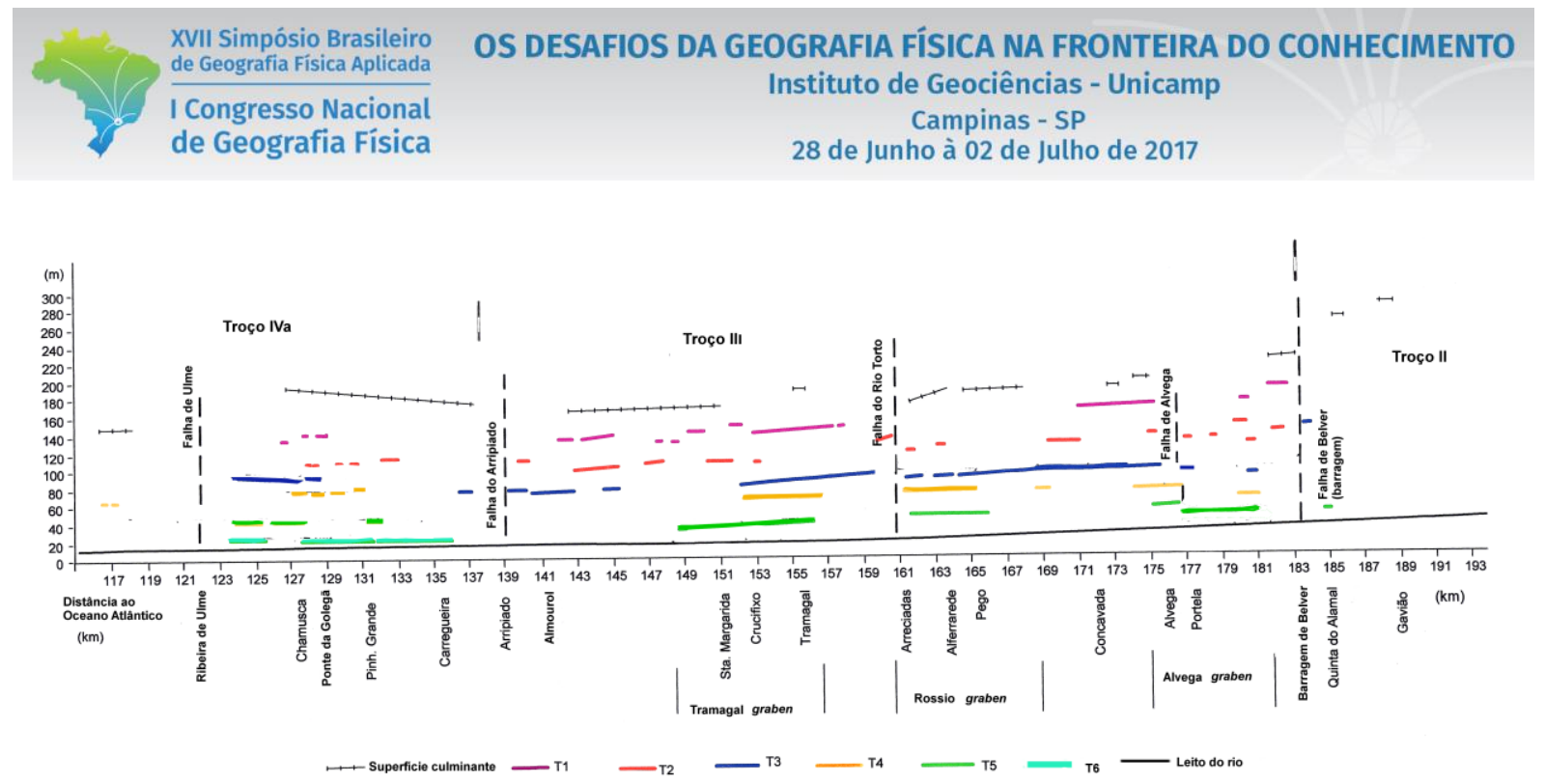

Fig. 2 - Perfil longitudinal do rio Tejo, junto à margem esquerda, abrangendo o troço III e parte dos troços adjacentes. Representa-se o leito atual, bem como a superfície dos terraços (T1 a T6) e da unidade sedimentar culminante. Observa-se que a unidade sedimentar culminante e os terraços estão localmente basculados e desnivelados por várias falhas (adaptado de Martins et al. 2009b).

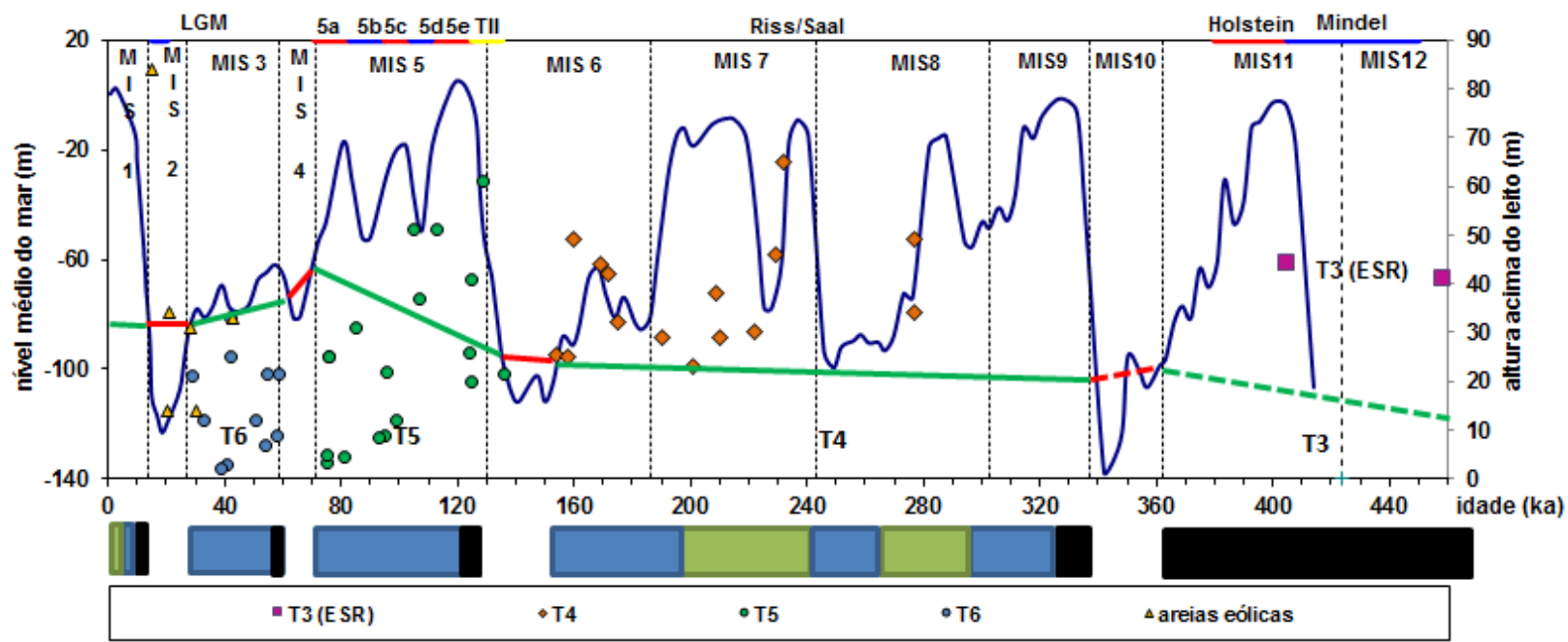

Fig. 3 - Curva de variação eustática do nível médio do mar (isótopos da V19-30, segundo Cutler et al. 2003) e projeção das idades (não mínimas) de LOE e ESR obtidas nos terraços T3 a T6 e Formação de Carregueira (areias eólicas) no sector inferior do Baixo Tejo (Cunha et al., 2016). As barras horizontais representam as litologias dominantes em cada unidade sedimentar (preto - cascalheira; azul - areia; verde - argila siltosa) e o provável intervalo de idade da agradação de cada terraço. Para os troços estudados, os prováveis períodos com espaço de acomodação estão representados pelos segmentos verdes e os períodos de incisão e alargamento do vale estão representados por segmentos de cor vermelha. Os intervalos dos MIS estão de acordo com Lisiecki \& Raymo (2005).

Caracterizaram-se oito unidades geomorfológicas/litostratigráficas que resultaram da evolução do rio Tejo em Portugal, desde a unidade culminante do enchimento sedimentar (SLD13) até ao leito actual. São 
enumeradas, da mais antiga para a mais recente (Cunha et al., 2016): uma unidade culminante do enchimento sedimentar (o ancestral Tejo, antes da etapa de incisão fluvial) - SLD13 (+142 a 262 m acima do leito actual; com provável idade 3,7 a 1,8 Ma), sem indústrias líticas identificadas; terraço T1 (+84 a $180 \mathrm{~m}$; ca. $1 \mathrm{Ma}$ a a $900 \mathrm{ka}$ ), sem indústrias; terraço T2 (+57 a $150 \mathrm{~m}$; idade do topo estimada em ca. 600 ka), sem indústrias; terraço T3 (+43 a 113 m; ca. 460 a 360? ka), sem indústrias; terraço T4 (+26 a 55 m; ca. 335 a 155 ka), Paleolítico Inferior (Acheulense) em níveis da base e intermédios mas Paleolítico Médio inicial em níveis do topo; terraço T5 (+5 a 34 m; 135 a 73 ka), Paleolítico Médio (com talhe Mustierense, Levallois); terraço T6 (+3 a 14 m; 62 a 32 ka), Paleolítico Médio final (Mustierense final); Areias da Carregueira (areias eólicas) e coluviões (+3 a ca. 100 m; 32 a 12 ka), Paleolítico Superior a Epipaleolítico; enchimento da planície aluvial (+0 a $8 \mathrm{~m}$; ca. 12 ka a actual), Mesolítico e indústrias mais recentes. As diferenças na elevação (a.r.b.) das escadarias de terraços resultam de soerguimento diferencial, devido a falhas ativas.

As idades dos terraços inferiores, atrás descritas, obtiveram-se através de diversos métodos de datação absoluta, que também possibilitaram estimar a idade do topo do terraço T2 (ca. $600 \mathrm{ka}$ ) e a provável idade do início da etapa de incisão (ca. 1,8 Ma). As idades permitiram constringir a fase de agradação dos terraços baixos e médios: T6 - 30 ka; T5 - 62 ka; T4 - ca. 180 ka; T3 - ca. 100? ka. Concluiu-se que durante o Plistocénico Médio e Final, no sector inferior do Baixo Tejo, a fase de incisão e alargamento do vale foram curtas (ca. 11-25 ka) e ocorreram durante períodos de nível do mar muito baixo, alternando com mais longas fases de inundação e de agradação do vale durante níveis do mar mais altos. Estas oscilações eustáticas de causa climática estão sobrepostas a um contexto de soerguimento de longo termo, controlando o desenvolvimento das escadarias.

Cunha et al. (2016) calculou que para os últimos ca. 155 ka as taxas de incisão de curto-termo apresentam valores $(0,09$ a $0,41 \mathrm{~m} / \mathrm{ka})$, aproximadamente, duplos dos calculados para o intervalo ca. 155 a $900 \mathrm{ka}$ $(0,04$ a $0,28 \mathrm{~m} / \mathrm{ka})$; este aumento na taxa de incisão deve estar relacionado com um aumento na taxa de soerguimento por intensificação da compressão devido à progressiva convergência entre as placas Africana e Eurasiática.

\section{Conclusões}

Os modernos estudos de Geomorfologia e de Geologia do Quaternário exigem a incorporação de idades absolutas para posicionar com precisão os eventos e quantificar processos. A datação por luminescência ou a por ESR determinam o tempo decorrido desde o último aquecimento ou exposição à luz do material; incertezas típicas: 5-10\% da idade. Têm uma grande aplicabilidade, dado necessitar de minerais geralmente omnipresentes e permitir datações desde poucas dezenas de anos a várias centenas de milhares 


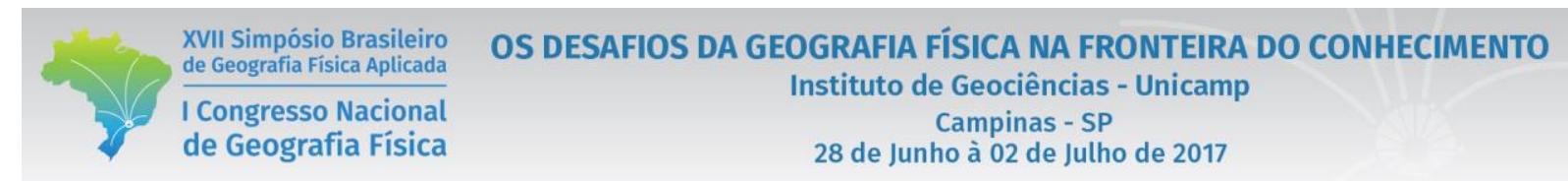

de anos (LOE) e desde algumas centenas de milhares de anos a alguns milhões de anos (ESR); facilmente aplicados na datação de cerâmicas, areias eólicas ou de praia, bem como a depósitos fluviais.

A amostragem deve ser convenientemente planeada e realizada com a colaboração de pesquisador com experiência na datação por LOE/ESR de sucessões sedimentares, e que também depois ajude na interpretação das idades obtidas e informe sobre os condicionalismos que as podem afectar. Uma adequada amostragem no campo é essencial para a obtenção de resultados concordantes com a estratigrafia e geomorfología. É necessário conhecer o contexto de enterramento e a história geológica ou as incertezas serão maiores.

Grãos incompletamente expostos à luz solar (ex. depósitos torrenciais, horizontes de solo, megalitos) podem ser datados, mas exigem medições em pequenos aliquotes e as incertezas serão maiores.

A datação em Qz pode permitir obter idades (não mínimas) até 140 ka em sedimentos com baixa taxa de radiação mas em sedimentos fluviais torna-se geralmente necessário usar o feldspato-K como dosímetro, tendo já sido possível datar os três mais recentes terraços do Tejo português, tendo-se alcançando idades até 300 ka.

Agradecimentos - Este estudo teve suporte financeiro pela Fundação para a Ciência e a Tecnologia e pela União Europeia no âmbito do COMPETE 2020 (Programa Operacional da Competitividade e Internacionalização), através dos projetos UID/MAR/04292/2013 - MARE e UID/GEO/04683/2013 ICT.

\section{Bibliografia}

BUYLAERT, J. P.; MURRAY, A. S.; THOMSEN, K. J.; JAIN, M. Testing the potential of an elevated temperature IRSL signal from K-feldspar. Radiation Measurements 44, 2009, p. 560-565.

BUYLAERT, J.-P.; JAIN, M.; MURRAY, A. S.; THOMSEN, K. J.; THIEL, C.; SOHBATI, R. A robust feldspar luminescence dating method for Middle and Late Pleistocene sediments. Boreas 41, 2012, p. 435-451.

CUNHA, P. P. Importância da datação por luminescência em estudos de Geomorfologia e de Geologia do Quaternário. Actas do V Congresso Nacional da Geomorfologia Portuguesa, 8-11 de Dezembro de 2010, Univ. Porto, organizado pela Associação Portuguesa de Geomorfólogos, 2010, p. 1-6. E-book (ISBN: 978-989-96462-2-3), http://www.apgeom.pt/Activid/e-book.pdf

CUNHA, P. P.; BUYLAERT, J.-P.; MURRAY, A. S.; ANDRADE, C.; FREITAS, M. C.; FATELA, F.; MUNHÁ, J. M.; MARTINS, A. A.; SUGISAKI, S. Optical dating of clastic deposits generated by an extreme marine coastal flood: the 1755 tsunami deposits in the Algarve (Portugal). Quaternary Geochronology, v. 5, Issues 2-3, 2010, p. 329-335.

CUNHA, P. P.; MARTINS, A. A.; DAVEAU, S.; FRIEND, P. Tectonic control of the Tejo river fluvial incision during the late Cenozoic, in Ródão - central Portugal (Atlantic Iberian border). Geomorphology 64, 2005. p. 271-298. 
CUNHA, P. P.; MARTINS, A. A.; HUOT, S.; MURRAY, A.; RAPOSO, L. Dating the Tejo river lower terraces in the Ródão area (Portugal) to assess the role of tectonics and uplift. Geomorphology 102, 2008. p. 43-54.

CUNHA, P. P.; ALMEIDA, N. A. C.; AUBRY, T.; MARTINS, A. A.; MURRAY, A. S.; BUYLAERT, J.-P.; SOHBATI, R., RAPOSO, L.; ROCHA, L. Records of human occupation from Pleistocene river terrace and aeolian sediments in the Arneiro depression (Lower Tejo River, central eastern Portugal). Geomorphology 165-166, 2012. p. 78-90.

CUNHA, P. P.; MARTINS, A. A.; GOUVEIA, M. P. As escadarias de terraços do Ródão à Chamusca (Baixo Tejo) - caracterização e interpretação de dados sedimentares, tectónicos, climáticos e do Paleolítico. Estudos do Quaternário, vol. 14, p. 1-24, 2016. http://www.apeq.pt/ojs/index.php/apeq

CUNHA, P. P; MARTINS, A. A.; BUYLAERT, J-P.; MURRAY, A.; RAPOSO, L.; MOZZI, P.; STOKES, M. New data on the chronology of the Vale do Forno sedimentary sequence (Lower Tejo River terrace staircase) and its relevance as a fluvial archive of the Middle Pleistocene in western Iberia. Quaternary Science Reviews, 2017. http://doi.org/10.1016/j.quascirev.2016.11.001

CUTLER, K. B.; EDWARDS, R. L.; TAYLOR, F. W.; CHENG, H.; ADKINS, J.; GALLUP, C. D.; CUTLER, P. M.; BURR, G. S.; BLOOM, A. L. Rapid sea-level fall and deep-ocean temperature change since the last interglacial period. Earth Planet. Sci. Lett. 206, 2003. p. 253-271.

HUNTLEY, D. J.; LIAN, O. B., Using optical dating to determine when a sediment was last exposed to sunlight. in: D.S. Lemmen and R.E. Vance (eds.), Holocene Climate and Environmental Change in the Palliser Triangle: A Geoscientific Context for Evaluating the Impacts of Climate Change on the Southern Canadian Prairies: Geological Survey of Canada Bulletin 534, 1999, p. 211-222.

LISIECKI, L. E.; RAYMO, M. E. A Pliocene-Pleistocene stack of globally distributed benthic $\delta 180$ records. Paleoceanography 20 (1), 2005. p. 1-17.

MARTINS, A. A.; CUNHA P. P. Vantagens e limitações da luminescência opticamente estimulada na datação de terraços do rio Tejo, sua importância na determinação da taxa de incisão fluvial. VII Congresso Nacional de Geologia (Resumos), Univ. Évora, 2006.

MARTINS, A. A.; CUNHA, P. P.; HUOT, S.; MURRAY, A.; BUYLAERT, J.-P. Geomorphological correlation of the tectonically displaced Tejo river terraces (Gavião-Chamusca area, Portugal) supported by luminescence dating. Quaternary International 199, 2009. p. 75-91.

MARTINS, A. A.; CUNHA, P. P.; BUYLAERT, J.-P.; HUOT, S; MURRAY, A. S.; DINIS, P.; STOKES, M.. KFeldspar IRSL dating of a Pleistocene river terrace staircase sequence of the Lower Tejo River (Portugal, western Iberia). Quaternary Geochronology 5 (2-3), 2010a. p. 176-180.

MARTINS, A. A.; CUNHA, P. P.; ROSINA, P.; OOSTERBECK, L.; CURA, S.; GRIMALDI, S.; GOMES, J.; BUYLAERT, J.-P.; MURRAY, A.S.; MATOS, J. Geoarchaeology of Pleistocene open air sites in the Vila Nova da Barquinha - Santa Cita area (Lower Tejo River basin, central Portugal). Proceedings of the Geologists' Association 121 (2), 2010b. p. 128-140.

MURRAY, A. S.; OLLEY, J. M. Precision and accuracy in the optically stimulated luminescence dating of sedimentary quartz: a status review. Geochronometria 21, 2002, p. 1-16.

MURRAY, A. S.; WINTLE, A. G. Luminescence dating of quartz using an improved single-aliquot regenerative-dose protocol. Radiaton Measurements, 32,2000, 57-73.

RAPOSO, L. Ambientes, territórios y subsistência en el Paleolítico Médio de Portugal. Complutum 6, 1995. p. 57-77.

ROSINA, P.; VOINCHET, P.; BAHAIN, J.; CRISTOVÃO, J.; FALGUÈRES, C. Dating the onset of Lower Tagus River terrace formation using electron spin resonance. Journal of Quaternary Science 29(2), 2014. p. 153-162.

WALlingA, J. Optically stimulated luminescence dating of fluvial deposits: a review. Boreas, 2002, p. 303322.

WINTLE, A. G., 1973. Anomalous fading of thermoluminescence in mineral samples. Nature, 245, p. 143-144. 


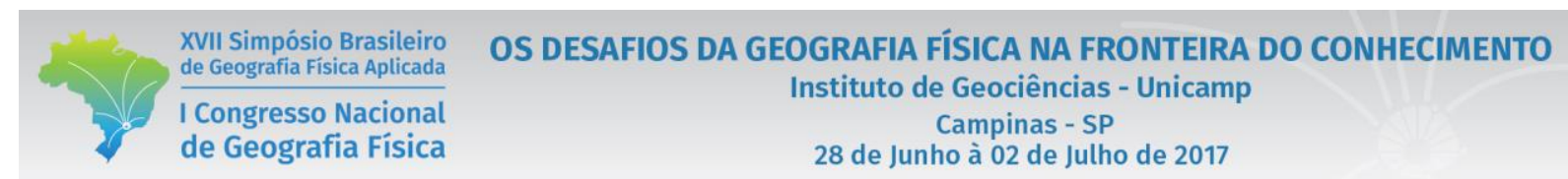

SOHBATI, R.; MURRAY, A.; BUYLAERT, J.-P.; ALMEIDA, N.; CUNHA, P. P.. Optically stimulated luminescence (OSL) dating of quartzite cobbles from the Tapada do Montinho archaeological site (eastcentral Portugal). Boreas 41 (3), 2012, p. 452-462. doi: 10.1111/j.1502-3885.2012.00249.x.

THOMSEN, K. J.; MURRAY, A. S.; JAIN, M.; BØTTER-JENSEN, L. Laboratory fading rates of various luminescence signals from feldspar-rich sediment extracts. Radiation Measurements 43, 2008, p. 1474-1486. 